\title{
A Novel Method to Predict Connectivity Probability of a Plane in Multi-Airline AANET
}

\author{
Xue Liu ${ }^{[1],[2]}$, Xiaoping Zeng ${ }^{[1]}$, Zhiming Wang ${ }^{[1]}$,Ya Wen ${ }^{[1]}$ and Bin Zhu ${ }^{[1]}$ \\ ${ }^{[1]}$ College of Communication Engineering, Chongqing University, Chongqing, \\ 400030, China; \\ ${ }^{[2]}$ Chongqing Communication Institute, Chongqing, 400035, China
}

\begin{abstract}
A novel method is introduced for approximate calculation of the probability that a plane can connect to a base station in an aeronautical ad hoc networks (AANET) where planes move along multiple specified airlines. Since the communication coverage of a plane can covers segments of airlines, for each of these segments, there is a probability that the communication of the plane (FCOM) can extend to it, from which FCOM can extend again to others segments of airlines until it reaches a base station. As multiple airlines are in AANET, there are plenty of extension paths for FCOM to reach a base station and each path has an existing probability, which is used to calculate the probability that the plane can connects to a base station. Thus, our method is in fact some kind of probabilistic diffusion, which works out the probability that the communication of a plane can diffuse to a base station. We choose the flight routes above the North Pacific Ocean and also generate some random scenarios in simulations to show the correctness and universality of our work. In the conclusion, several future works of AANET based on our method are introduced.
\end{abstract}

Keywords: AANET, Multi-airline, connectivity, probabilistic diffusion

\section{Introduction}

With the rapid development of civil aviation, the demands of accessing Internet on board have become stronger than ever before. However, in polar, desert, marine and other areas where base stations can't be built, aircrafts have to use satellite links or large span multi-hop links within AANET to reach base stations. Due to the large delay, high cost and limited bandwidth of satellite links, AANET is preferred for Internet accessing in these regions [1-2].

For AANET, the Newsky project has proposed networking strategies with mobile IPv6 technology [3]. ATENAA project has studied Ka-band array antennas and optical communications between aircrafts [4]. Justin R, Abdul $\mathbf{J}$ et al have presented cross-layer networking solutions among physical, mac, network and transport layers [5]. Quentin Vey et al have proposed the use of Direct Sequence CDMA (DS-CDMA) at the access layer [2] while Jianshu Yan et al have studied the capacity of single flight path AANET [9].

Among all the topics on AANET, connectivity analysis of networks is one of the most fundamental issues [1-2]. So far, Ehssan S, Daniel M, Zheng Bo, TXM Nguyen, D Medina et al have done several valuable works. The methods they employed can be classified into two categories. The first is experimental method, which uses the real flight data about a specified airspace to analyze the connectivity $[2,6]$. It acquires accurate result, but lacks universality. The second is theoretical method, which is based on the features of Poisson point process to calculate the connectivity probability, but all of their works are only dealing with single airline or parallel airlines[7-9] and, to our best knowledge, no work is about multiple crossing airlines. 
In fact, the connectivity problem of mobile ad-hoc networks has been widely discussed, but most of them are focused on one-dimensional scenario [10-12] or two-dimension where nodes move randomly in a plane [13-15] not along multiple specified lines. Though nodes in vehicle ad-hoc networks(VANET) are move along specified lines like AANET, in most cases, their works focus on vehicles' connectivity in a high way[16], and it is a "single line" scenario too. For "multi-line" in VANET, vehicles must be on city roads. In that scenario, the speed of vehicles can be affected by plenty of factors, such as pedestrian crosswalks, traffic jams, traffic lights and so on. Thus, the probability distribution of the number of vehicles in a road is hard to assume and the theoretical calculation of the connectivity probability of vehicle to vehicle in multi-road VANET is believed to be very complex.

Therefore, the connectivity problem of mobile ad-hoc networks in which nodes move along multiple specified lines has not been solved so far and we give a method to approximately calculate the probability that a plane can connect to a base station in multi-airline AANET.

\section{Mathematical Method}

Since AANET is not deployed currently and none of the achievements mentioned in paragraph two of previous section is taken as standard or in real use, we don't consider the physical and access layers of AANET and use a fixed value, $R$, as the transmission radius for all planes, which is around $500 \mathrm{~km}$ (nearly $300 \mathrm{nmi}$ as used in reference [6]).

According to the specifications of the International Civil Aviation Organization (ICAO), the airspace is divided into several height levels. The distance between two height levels is around $500 \mathrm{~m}$ and the maximum height of a civil airline is around $10 \mathrm{~km}$, which are both far less than the assumed transmission radius of a plane. Therefore, we ignore the interval length of two airlines and the vertical distance between airline and base station in our work. Moreover, as the radius of earth is far greater than $R$, airlines are looked as straight lines. Then, in Figure 1, we draw several airlines and base stations in a two dimensional scenario, in which $H_{i}$ stands for airline $i(i=1,2,3 \ldots)$ and $B S_{k}$ denotes base station $k(k=1,2,3 \ldots)$, and the spatial distribution of planes in each airline is assumed to follow Poisson point process with density $\rho_{i}$ for $H_{i}$.

Before introducing our method, we define $C C_{x}$ as the communication coverage of point $x$, which is a circle centered at point $x$ with radius $R$, and $C C_{x y}$ as the communication coverage of segment $x y$, which is formed by all the circles centered at the points in segment $x y$ with radius $R$ (like area $S_{2}$ in Figure 1).

In Figure 1, a plane (call it $F$ ) is at point $w$ in $H_{1}$ and its communication coverage is $C C_{w}\left(\right.$ area $S_{1}$ ). As seen, no base station is in $C C_{w}$, so the connection between $F$ and a base station needs the help of other planes in the airlines. Since segment $a b$ is covered by $C C_{w}$, the planes in $a b$ can be used to establish the connection between $F$ to a base station. That means the communication of $F$ (FCOM) can extend to $a b$ with a probability. After the extension, $C C_{w}$ changes to $C C_{a b}\left(\right.$ area $\left.S_{2}\right)$ because plane may be at any point in ab. Then, FCOM can extend from $a b$ to $c d$ with another probability. Finally, a base station is under $C C_{c d}$ and there is also a probability that FCOM can reach $B S_{3}$ from $c d$.

Let $P_{\text {start }}$ (First-hop Probability) denote the probability that FCOM extends from point $f$ to $a b, P_{a b \rightarrow c d}$ (Transition Probability) denote the probability that FCOM extends from $a b$ to $c d$ and $P_{\text {last }}$ (Last-hop Probability) denote the probability that FCOM extends from $c d$ to $B S_{3}$. Then, the probability that FCOM can reach a base station through the path $\left(w \rightarrow a b \rightarrow c d \rightarrow B S_{3}\right.$ ) is denoted by $P_{\text {path }}$, which equals $P_{\text {start }} \times P_{a b \rightarrow c d} \times P_{\text {last }}$. Apparently, there are more than one path, e.g., FCOM extends to base station $B S_{1}$ through airline $H_{1}$ or 
$B S_{2}$ through airline $\mathrm{H}_{2}$.

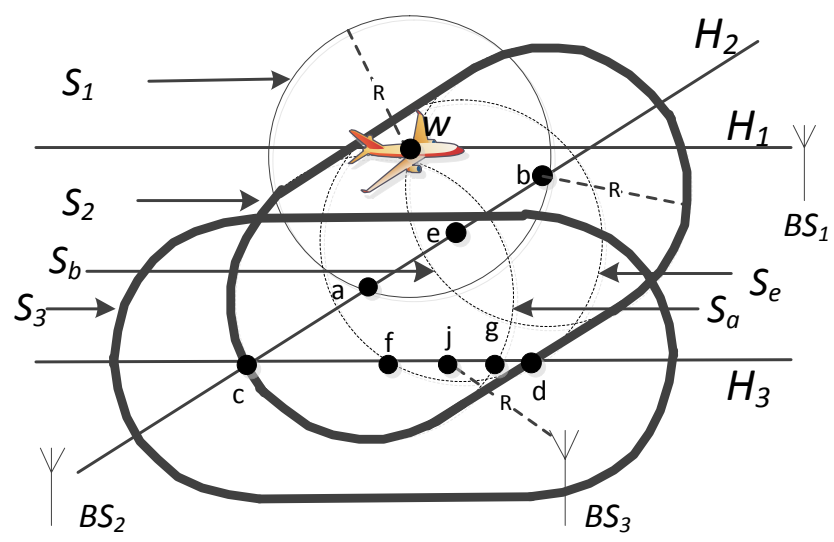

Figure 1. A Multi-Airline Scenario

In fact, the probability of each path may contain any number of Transition Probabilities, which means multiple extensions may be needed for FCOM to reach a base station. In the Part-A of this section, two of the FCOM's extension paths are found manually according to Figure 1 to introduce our method and all the paths are found through an algorithm in section III, which is suitable for any scenario.

\subsection{Connectivity Probability of Extension Path}

By defining $l_{x y}$ as the length of segment $x y$ and $P_{x y}(K)$ as the distribution function of the number of planes on segment $x y$, we give the First-hop Probability in Formula 1 according to the property of Poisson point process, which equals the probability that at least one plane is in segment $a b$.

$$
P_{\text {start }}=P_{a b}(K \geq 1)=1-e^{-l_{a b} \rho_{2}}
$$

The Transition Probability $P_{a b \rightarrow c d}$ is the product of the probabilities that at least one plane is in segment $c d$ and that $c d$ is covered by $S_{2}$. Since a plane may be at any point in $a b$ and the probability that it exists at a specified point is infinitesimal, $S_{2}$ is formed by infinitely many circular regions and each region has an infinitesimal coverage probability (ICP). Therefore, each circular region intersecting with segment $c d$ covers a short part of $c d$ with an ICP and it is complicated to work out the Transition Probability with these ICPs. To make it simpler, this paper distributes the ICPs, the sum of which must be "1" in the condition that FCOM has extended to $a b$, into limited number of circular regions in $S_{2}$. The coverage probability (CP) of each new circular region is a real number and every new circular region may cover a part of $c d$ too.

In the following context, we distribute all ICPs into only three circular regions to give an approximate calculation. However, the ICPs can be allocated to more circular regions by using the same method to gain higher precision.

These three circular regions, $S_{a}, S_{b}$ and $S_{e}$ as shown in Figure 1, are centered at the two endpoints and the midpoint $e$ of segment $a b$ respectively and their CPs are denoted by $P_{a}, P_{b}$ and $P_{e}$ respectively. The sum of $P_{a}, P_{b}$ and $P_{e}$ should be "1" by ignoring the extremely small CP of the area in $S_{2}$, which is not covered by $S_{a}, S_{b}$ or $S_{e}$. After the distribution, segment $c g$ is covered by $S_{a}$ while $f d$ is covered by $S_{e}$. Apparently, $C C_{c g}$ and $C C_{f d}$ are both covering $B S_{3}$. Thus, FCOM has at least two extension paths to $B S_{3}$, the one is $w \rightarrow a b \rightarrow c g \rightarrow B S_{3}$ and the other is $w \rightarrow a b \rightarrow f d \rightarrow B S_{3}$. Connectivity 
probabilities through these two paths are denoted by $P_{\text {path } 1}$ and $P_{\text {path } 2}$ respectively in Formula 2 and Formula 3.

$$
\begin{aligned}
& P_{\text {path } 1}=P_{\text {start }} \times P_{a b \rightarrow c g} \times P_{\text {last } 1} \\
& P_{\text {path } 2}=P_{\text {start }} \times P_{a b \rightarrow f d} \times P_{\text {last } 2}
\end{aligned}
$$

$P_{a b \rightarrow c g}$ and $P_{a b \rightarrow f d}$ are Transition Probabilities which are equal to $P_{a} \times P_{c g}(K \geq 1)$ and $P_{e} \times P_{f d}(K \geq 1)$ respectively. $P_{\text {lass } 1}$ and $P_{\text {lass } 2}$ are the Last-hop Probabilities. Since planes distribute uniformly in each airline, which is also a property of Poisson point process, the Last-hop Probability is a ratio of two segments' length. The denominator of the ratio is the length of the last segment reached by FCOM and the numerator is the length of the last segment's sub-segment in whose communication coverage there is a base station. Thus $P_{\text {las } 1}$ and $P_{\text {last } 2}$ are equal to $l_{j g} / l_{c g}$ and $l_{j d} / l_{f d}$ respectively.

If there is no base station under the coverage of $c g$ or $f d$, FCOM can extend separately from $c g$ or $f d$ to other segments until reaching a base station.

\subsection{Calculation of $P_{e}, P_{a}$ and $P_{b}$}

Since each circular region centered on segment $a b$ must have an overlapping region with $S_{e}$, we let $S_{\text {esum }}$ denote the sum of the areas of the overlapped regions. Then, by defining $P_{e}^{\prime}$ as the probability that the communication coverage drops into $S_{e}$ after FCOM extending to segment $a b$, we obtain Formula 4.

$$
P_{e}^{\prime}=\frac{S_{\text {esum }}}{\text { the sum of the areas of all circular regions centered on } a b}
$$

Since the numerator and denominator in Formula 4 are both infinite, an approximate calculation is needed. In Fig 2, $l$ denotes the length of segment $a b$, which is divided into many $\Delta x$, and at every $\Delta x$ there is a circle with radius $R$ centered at it. Points $a$, $e$ and $b$ are at the origin, $l / 2$ and $l$ respectively. Each circular region which is centered at integral multiple of $\Delta x$ must have an overlapping region with $S_{e}$ and the shadow section shows one of the overlapping regions. The total areas of these intersection regions, $S_{\text {esum }}$, can approximately represented by the numerator of Formula 5, the denominator of which is an approximation of the sum of the areas of all circular regions centered on $a b$ mentioned in Formula 4. Thus $P_{e}^{\prime}$ is approximated by Formula 5.

$$
P_{e}^{\prime} \sim \frac{2 \sum_{n=0}^{l /(2 \Delta x)}\left(2 R^{2} \cos ^{-1} \frac{l-2 n \Delta x}{8 R}-\frac{l-2 n \Delta x}{4} \sqrt{R^{2}-\frac{(l-2 n \Delta x)^{2}}{64}}\right)}{\pi R^{2} l / \Delta x}
$$

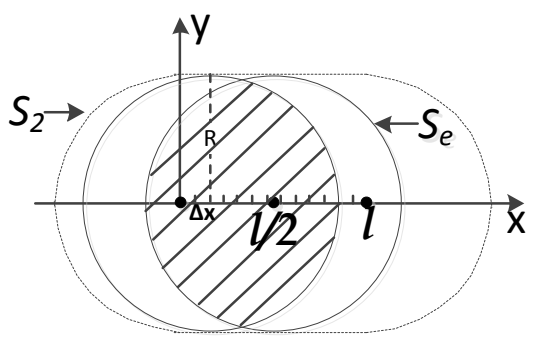

Figure 2. ICPS Distribution

By multiplying $\Delta x$ to both the numerator and denominator in Formula 5 and limiting $\Delta x$ close to zero, $P_{e}^{\prime}$ is worked out in Formula 6: 


$$
\begin{gathered}
P_{e}^{\prime}=\frac{2 \int_{0}^{l / 2}\left(2 R^{2} \cos ^{-1} \frac{l-2 x}{4 R}-\frac{l-2 x}{2} \sqrt{R^{2}-\frac{(l-2 x)^{2}}{16}}\right) d x}{\pi R^{2} l} \\
=\frac{G_{1}-G_{2}}{\pi R^{2} l} \\
G_{1}=8 R^{3}\left(\frac{l}{4 R} \cos ^{-1} \frac{l}{4 R}-\sqrt{1-\frac{l^{2}}{16 R^{2}}}+1\right) \\
G_{2}=\frac{8}{3}\left[R^{3}-\left(R^{2}-\frac{l^{2}}{16}\right)^{3 / 2}\right]
\end{gathered}
$$

In the same way, $P_{a}^{\prime}$ can be calculated in Formula 7 and $P_{b}^{\prime}$ equals $P_{a}^{\prime}$.

$$
\begin{gathered}
P_{a}^{\prime}=\frac{G_{3}-G_{4}}{\pi R^{2} l} \\
G_{3}=4 R^{3}\left(\frac{l}{2 R} \cos ^{-1} \frac{l}{2 R}-\sqrt{1-\frac{l^{2}}{4 R^{2}}}+1\right) \\
G_{4}=\frac{4}{3}\left[R^{3}-\left(R^{2}-\frac{l^{2}}{4}\right)^{3 / 2}\right]
\end{gathered}
$$

Since $S_{e}, S_{a}$ and $S_{b}$ are always partially overlapped, the CP of the overlapping region should be shared. We define $P_{u v}^{\prime}(u$ only stands for point $e, a$ or $b$ and so does $v)$ as the probability that the communication coverage drops into the overlapping region of $S_{w}$ and $S_{v}$ after FCOM extending to segment $a b$ and let $P_{a b e}^{\prime}$ denote the probability that the communication coverage drops into the overlapping region of $S_{e}, S_{a}$ and $S_{b}$ after the same extension of FCOM. Then, in the same way we calculate $P_{e}^{\prime}$, the expressions of $P_{e a}^{\prime}, P_{e b}^{\prime}, P_{a b}^{\prime}$ and $P_{a b e}^{\prime}$ are given in Formulas 8, 9, 10 and 11 respectively.

$$
\begin{gathered}
P_{e a}^{\prime}=\frac{G_{5}+G_{6}-G_{7}}{\pi R^{2} l} \\
G_{5}=R^{2} l \cos ^{-1} \frac{l}{4 R}-\frac{l^{2}}{4} \sqrt{R^{2}-\frac{l^{2}}{16}} \\
G_{6}=4 R^{3}\left(\frac{l}{2 R} \cos ^{-1} \frac{l}{2 R}-\sqrt{1-\frac{l^{2}}{4 R^{2}}}-\frac{l}{4 R} \cos ^{-1} \frac{l}{4 R}+\sqrt{1-\frac{l^{2}}{16 R^{2}}}\right) \\
G_{7}=\frac{4}{3}\left[\left(R^{2}-\frac{l^{2}}{4}\right)^{3 / 2}-\left(R^{2}-\frac{l^{2}}{16}\right)^{3 / 2}\right] \\
P_{a b}^{\prime}=\frac{2 R^{2} l \cos ^{-1} \frac{l}{2 R}-l^{2} \sqrt{R^{2}-\frac{l^{2}}{4}}}{\pi R^{2} l} \\
P_{a b e}^{\prime}=\frac{2 R^{2} l \cos ^{-1} \frac{l}{2 R}-l^{2} \sqrt{R^{2}-\frac{l^{2}}{4}}}{\pi R^{2} l}
\end{gathered}
$$

After the calculation for the overlapping regions, we share the probabilities that the communication coverage drops into the overlapping regions and get $P_{e}^{\prime \prime}, P_{a}^{\prime \prime}$ and $P_{b}^{\prime \prime}$ in Formulas 12, 13 and 14 respectively, which will be used in the expressions of $P_{e}, P_{a}$ and $P_{b}$. 


$$
\begin{aligned}
& P_{e}^{\prime \prime}=P_{e}^{\prime}-\frac{P_{e a}^{\prime}}{2}-\frac{P_{e b}^{\prime}}{2}+\frac{P_{a b e}^{\prime}}{3} \\
& P_{a}^{\prime \prime}=P_{a}^{\prime}-\frac{P_{e a}^{\prime}}{2}-\frac{P_{a b}^{\prime}}{2}+\frac{P_{a b e}^{\prime}}{3} \\
& P_{b}^{\prime \prime}=P_{b}^{\prime}-\frac{P_{a b}^{\prime}}{2}-\frac{P_{e b}^{\prime}}{2}+\frac{P_{a b e}^{\prime}}{3}
\end{aligned}
$$

Since $S_{e}, S_{a}$ and $S_{b}$ can't cover the full area of $S_{2}$ and the uncovered area is very small, the sum of $P_{e} ", P_{a} "$ and $P_{b} "$ is always slightly less than 1 . By normalizing them, we work out $P_{e}, P_{a}$ and $P_{b}$ through Formulas 15, 16 and 17 respectively.

$$
\begin{aligned}
& P_{e}=\frac{P_{e} "}{P_{e} "+P_{a} "+P_{b} "} \\
& P_{a}=\frac{P_{a} "}{P_{e} "+P_{a} "+P_{b} "} \\
& P_{b}=\frac{P_{b} "}{P_{e} "+P_{a} "+P_{b} "}
\end{aligned}
$$

\subsection{FCOM's Extension along its own Airline}

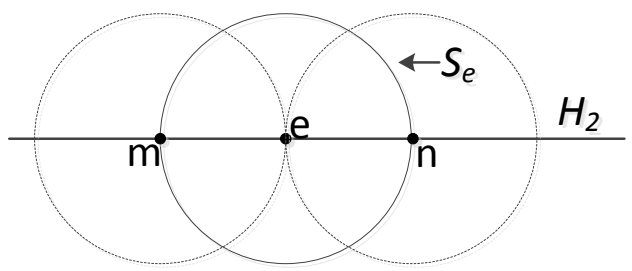

Figure 3. FCOM's Extension Along The Same Airline

FCOM's extension to different airlines is elaborated above and it can also extend along its own airline in the same way. For example, the circular region $S_{e}$, as shown in Figure 3 , covers the segment $m n$ of its own airline $H_{2}$. The communication coverage of segment $m n, C C_{m n}$, is also formed by infinitely many circular regions and each region has an infinitesimal coverage probability(ICP) too. Thus, in the same way as we deal with the ICPs of $C C_{a b}$ in Part A of this section, the ICPs of $C C_{m n}$ are distributed into three circular regions too and we call them $S_{e}^{\prime}, S_{m}$ and $S_{n}$, which are centered at points $e$, $m$ and $n$ respectively. Since $S_{e}^{\prime}$ and $S_{e}$ are the same regions, the ICPs is only distributed equally to $S_{m}$ and $S_{n}$ to avoid repeated calculation and each of them shares 0.5 .

\section{Algorithm for Finding Extension Paths}

Given the coordinates of base stations, line equations of airlines and plane densities of airlines, the algorithm in Table.1 finds all the FCOM's extension paths which are from a plane at point $A\left(A_{x}, A_{y}\right)$ in airline $z$ to one of the base stations.

The function "Function_Probability" in Table 1 starts with parameters $C x=A x, C y=A y, P=1, P_{\text {temp }}=1$ and $Z=z$. The First-hop Probability is not mentioned in the function, because it equals the Transition Probability when $P_{\text {temp }}$ is 1 . From lines 10 to 24 of the function, we deal with FCOM's extension to different airlines while lines 24 to 34 are for the extension along its own airline. Since the probabilistic 
diffusion of FCOM can go in any direction, its extension paths can be looked as independent ones. Thus, assuming the total number of the Table 1.

Function For Connectivity Probability Calculation

Function_Probability $\left(C_{x}, C_{y}, P, P_{\text {temp }}, Z\right)$

Compute $l_{i}$, which stands for the length of the segment on airline $\mathrm{i}$ covered by $S^{(1)}$. If airline $\mathrm{i}$ doesn't pass through $S, \quad l_{i}$ equals zero.

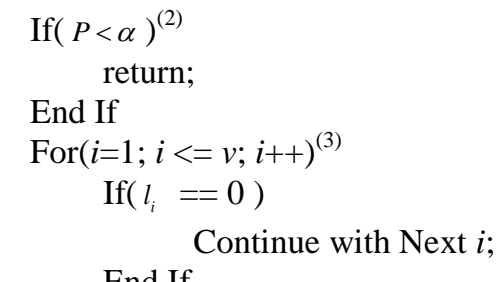

Line 10: $\quad$ If $(i !=Z)$

$P_{h i}(K \geq 1)=1-e^{-l_{i} \rho_{i}}(4,5)$;

Calculate Transition Probability:

$P_{h z->h i}=P_{\text {temp }} \times P_{h i}(K \geq 1)$

If(a base-station is covered by $\left.E_{i}\right)^{(6)}$

Compute the Last-hop Probability $P_{\text {last }}$;

Output $P_{\text {out }}=P \times P_{h z \rightarrow h i} \times P_{\text {last }}$

return;

End If

Compute the coordinates of point $a\left(a_{x}, a_{y}\right), b\left(b_{x}, b_{y}\right)$ and $e\left(e_{x}, e_{y}\right)^{(7)}$;

Allocate the coverage probability of $E_{i}$ to $P_{a}, P_{b}$ and $P_{e}{ }^{(8)}$;

Divide one path into three:

Function_Probability $\left(a_{x}, a_{y}, \quad P \times P_{h z \rightarrow h i}, P_{a}, i\right)$;

Function_Probability $\left(b_{x}, b_{y}, \quad P \times P_{h z \rightarrow h i}, P_{b}, i\right)$;

Function_Probability $\left(e_{x}, e_{y}, P \times P_{h z \rightarrow h i}, P_{e}, i\right)$;

Line 24: Else

If(a base-station is covered by $\left.E_{z}\right)^{(6)}$

Compute the Last-hop Probability $P_{\text {last }}$;

Output $P_{\text {out }}=P \times P_{\text {temp }} \times P_{\text {last }}$;

return;

End If

Compute the coordinates of $m\left(m_{x}, m_{y}\right)$ and $n\left(n_{x}, n_{y}\right)^{(9)}$;

Divide one path into two:

Function_Probability $\left(m_{x}, m_{y}, P \times P_{\text {temp }}, 0.5, Z\right)$;

Function_Probability $\left(n_{x}, n_{y}, P \times P_{\text {temp }}, 0.5, Z\right)$;

Line 34: End If

End For

End Function_Probability

Annotations for the function: (1) $S$ is the circular region centered at point $C\left(C_{x}, C_{v}\right)$ with radius $R$; (2) If $P$ is too small, it is abandoned to keep the algorithm fast and the threshold $\alpha$ is set to 0.0001 in this paper; (3) $v$ denotes the number of airlines; (4) $\rho_{i}$ is the plane density on airline $i$; (5) hi denotes the segment on airline $i$ covered by $S$ and $P_{h i}(K)$ is probability distribution of plane number on segment $h i$; (6) $E_{i}$ denotes the communication coverage of $h i$ and we assume only one base-station can be covered by $E_{i}$ simultaneously; (7) Points $a, b$ and $e$ are the two endpoints and midpoint of $h i$ respectively; (8) $P_{a}, P_{b}$ and $P_{e}$ are calculated in the same way as mentioned in section II; (9) Points $m$ and $n$ are crossing points of $S$ and airline $Z$. 
extension paths is $n$, defining $P_{i}(i=1,2,3 \ldots n)$ as the existing probability of path $i$ and with the help of the addition formula of probability theory ${ }^{[17]}$, we work out the probability that a plane at point $A\left(A_{x}, A_{y}\right)$ can connect to a base station, which is denoted by $P_{A x, A y}$ in Formula 18

$$
P_{A x, A y}=\sum_{i=1}^{n} P_{i}-\sum_{1 \leq i<j \leq n} P_{i} P_{j}+\sum_{1 \leq i<j<k \leq n} P_{i} P_{j} P_{k}+\cdots+(-1)^{n-1}\left(P_{1} P_{2} \cdots P_{n}\right)
$$

\section{Simulation}

In simulation, we first choose the flight routes above the North Pacific Ocean drawn in Figure 4, which is acquired from www.flightaware.com, to show the correctness of our work. Then we generate some random scenarios to show the universality of our method.

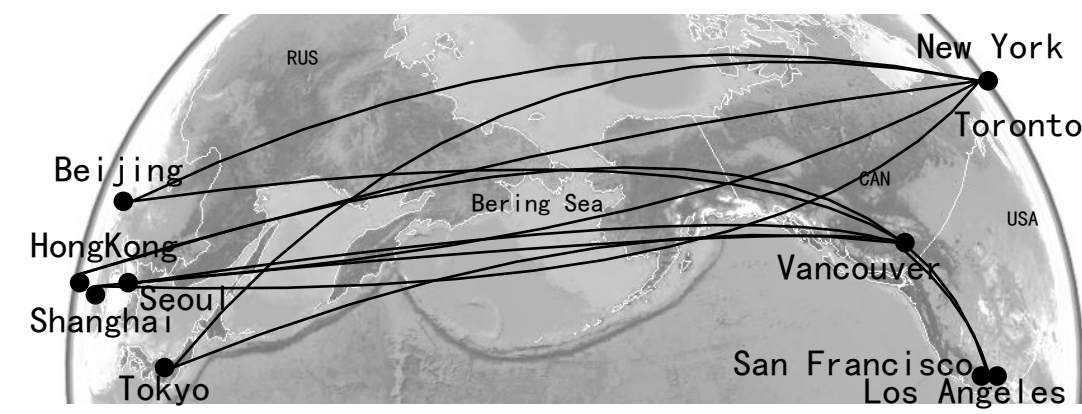

Figure 4. Routes above North Pacific Ocean

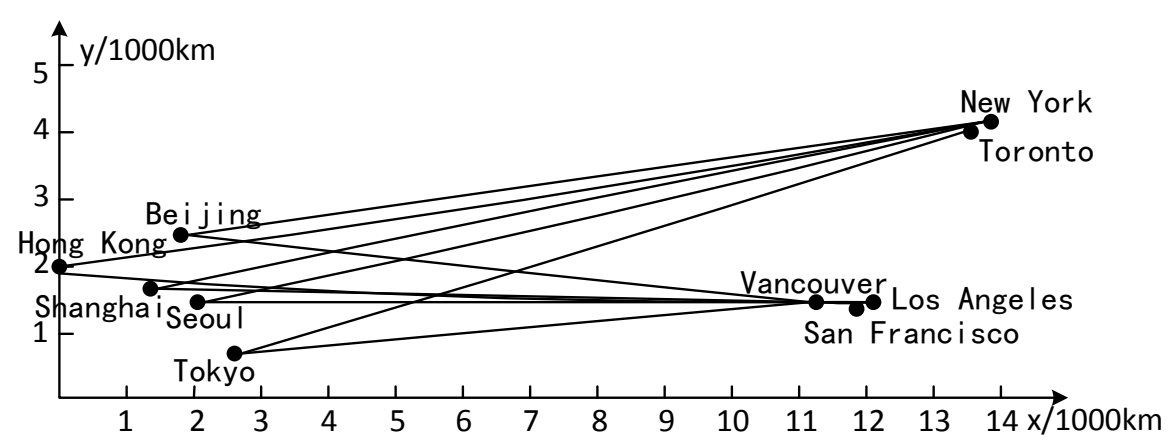

Figure 5. Routes for Simulation

Table 2. Routes and Average Plane Density

\begin{tabular}{|c|c|c|c|}
\hline $\begin{array}{c}\text { Two-Way } \\
\text { Routes }\end{array}$ & $\begin{array}{l}\text { Plane } \\
\text { number }\end{array}$ & $\begin{array}{c}\text { Average } \\
\text { plane number }\end{array}$ & $\begin{array}{l}\text { Density } \\
(/ 1000 \mathrm{~km})\end{array}$ \\
\hline HongKong--LA\&SF\&Van & 10 to 16 & 13.6 & 1.208 \\
\hline Shanghai--LA\&SF\&Van & 6 to 9 & 7.8 & 0.788 \\
\hline Beijing--LA\&SF\&Van & 5 to 11 & 7.8 & 0.821 \\
\hline HongKong--NY & 8 to 12 & 9.7 & 0.694 \\
\hline Shanghai-NY & 3 to 5 & 4.4 & 0.347 \\
\hline Beijing--NY & 6 to 8 & 7.5 & 0.619 \\
\hline Seoul--LA\&SF\&Van & 3 to 22 & 12.9 & 1.395 \\
\hline Seoul--NY & 12 to 18 & 15.1 & 1.249 \\
\hline Tokoy--LA\&SF\&Van & 5 to 15 & 10.7 & 1.232 \\
\hline Tokoy--NY & 9 to 11 & 9.9 & 0.845 \\
\hline
\end{tabular}

According to the explanations in paragraph two of section II, Figure 4 are mapped from $3 \mathrm{D}$ to $2 \mathrm{D}$ in Figure 5, where New York and Toronto are set in the same routes because 
they are so close and so are Vancouver, Los Angeles and San Francisco. On each route, the plane number is various at different times of the day and we choose the peak hours for simulation and theoretical calculation, which are from 15:00 to 21:00 in Beijing time. Plane number and density (plane number per $1000 \mathrm{~km}$ ) for each route in peak hours are shown in Table 2. A base station is set at each endpoint of every route and the transmission radius of a plane, $R$, is set to $500 \mathrm{~km}$.

In the route of Shanghai to Vancouver, 100 value points are set along the route and they are uniformly-spaced. For each value point, we calculate the theoretical probability that the plane at that value point can connect to a base station. Then, we use Matlab to generate planes on each airline according to Poisson point process with the densities in Table. 2 to get simulation result.

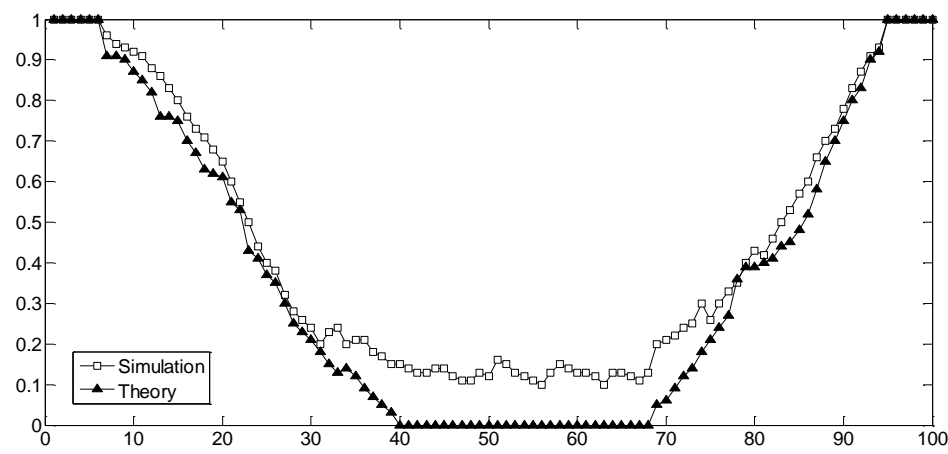

Figure 6. Theoretical and Simulation Values

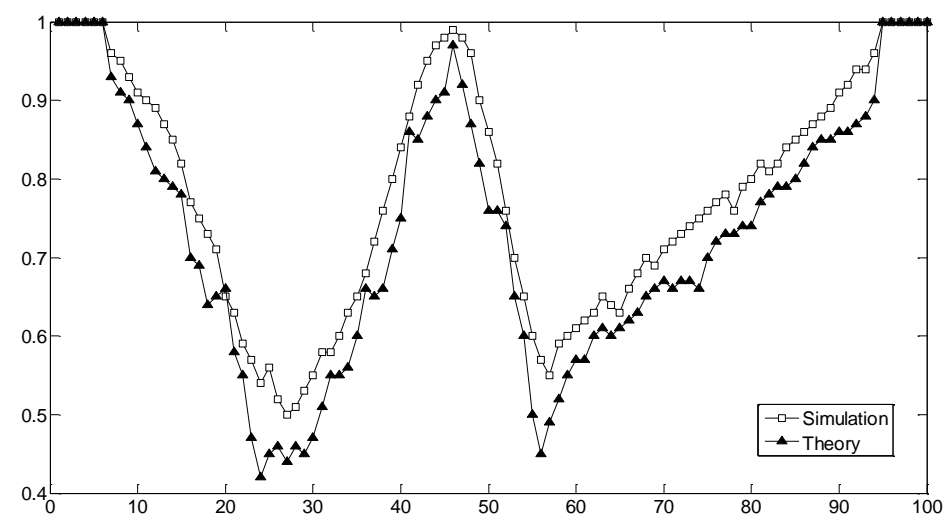

Figure 7. Probability Values with more Base Stations

Once a plane passing through a value point, a set of data is recorded, which includes the information of all planes' locations at that time. For each value point, we take 100,000 sets of data and record the count of how many times that the plane at that value point can connect to a base station. Then, we calculate the ratio of the count to 100,000 to get the simulation result. As shown in Figure 6, the theoretical value is close to the simulation result. However, when the theoretical value is smaller than 0.1 , there is an obvious deviation between them. That is because, when the probability of a path is too small, it is abandoned as mentioned in "Function_Probability" in section III to keep the algorithm fast.

As seen, most connectivity probabilities in Figure 6 are too small, to increase them, we add two new base stations at the coordinate $(6,2.3)$ and $(8.5,1)$ in Figure 5 , which are near Bering Sea and in CAN respectively. Then, the theoretical calculation and the simulation are made again and their results are shown in Figure 7, 
To verify the universality of our method, we generate three more random scenarios in Figure 8, Figure 9 and Figure 10 respectively. For each scenario, we choose the airline marked with "AIRLINE" to set value points and mark the base stations with white points of black edge. The numbers of the base stations are 8,7 and 8 for random scenarios 1,2 and 3 respectively. All the plane densities of the airlines are set to $3 / 1000 \mathrm{~km}$ and $R$ is set to $350 \mathrm{~km}$ in all the three scenarios. Then, for all the value points on the marked airlines(from the left end point to the right end point of the airline), we give the connectivity probabilities for scenarios 1, 2 and 3 respectively in Figure 11, Figure 12 and Figure 13.

As seen, in all the figures about numeric results in this section, there are always some deviation between theoretical value and simulation value. To reduce the deviation, we can distribute the ICPs into more than three circular regions and it is believed that the theoretical value will be more precise. However, the complexity of our algorithm is also increased along with the increase of the circular regions.

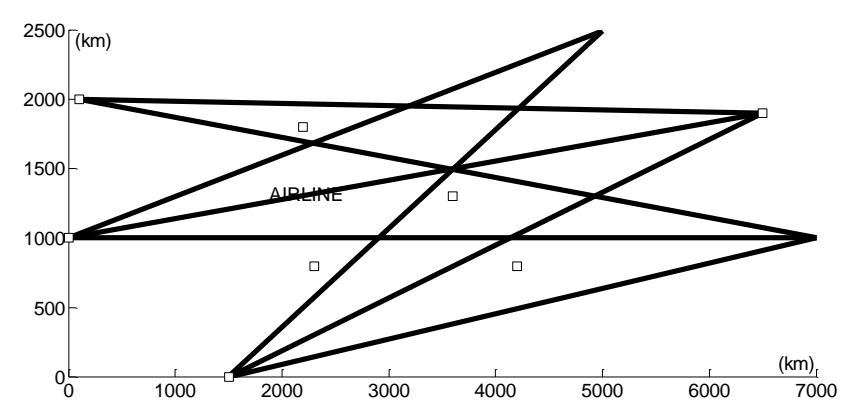

Figure 8. Random Scenario 1

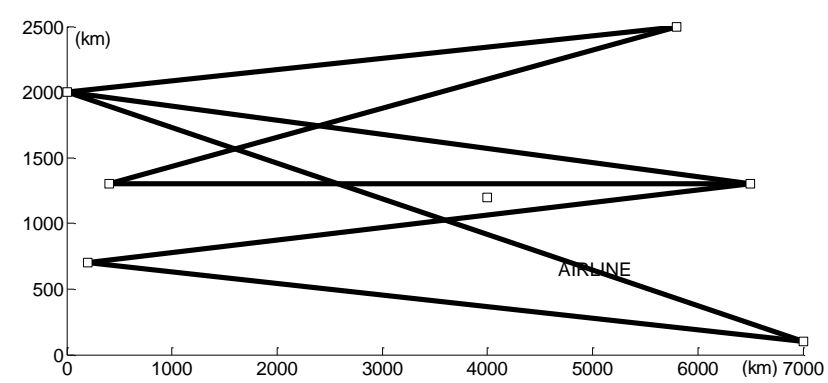

Figure 9. Random Scenario 2

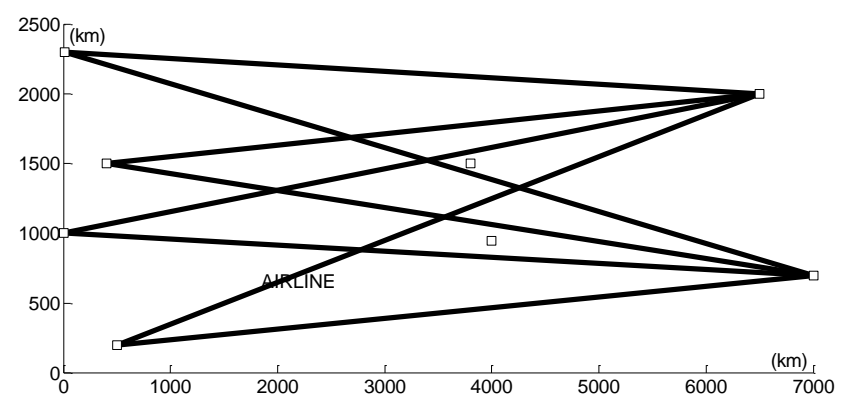

Figure 10. Random Scenario 3 


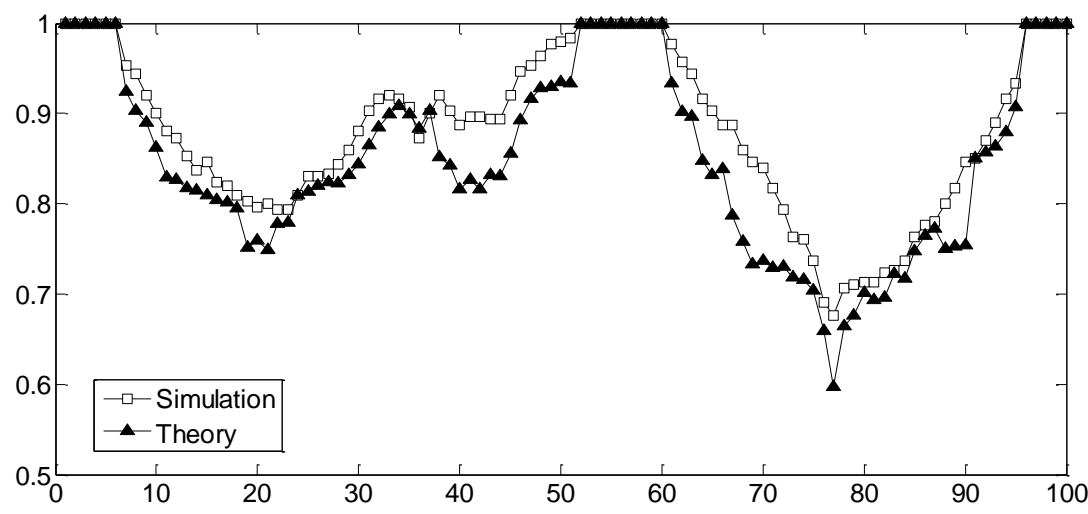

Fig 11. Numeric Result For Random Scenario 1

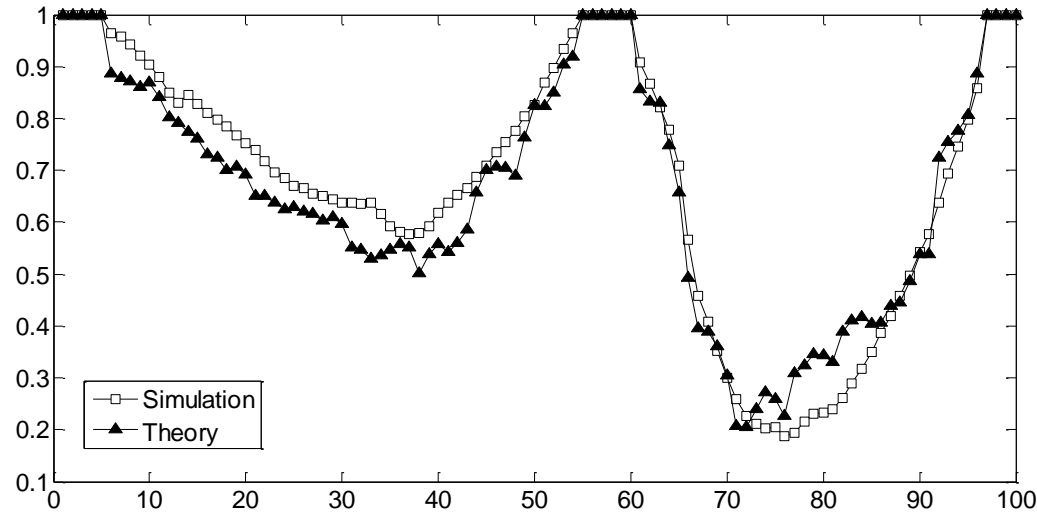

Figure 12. Numeric Result For Random Scenario 2

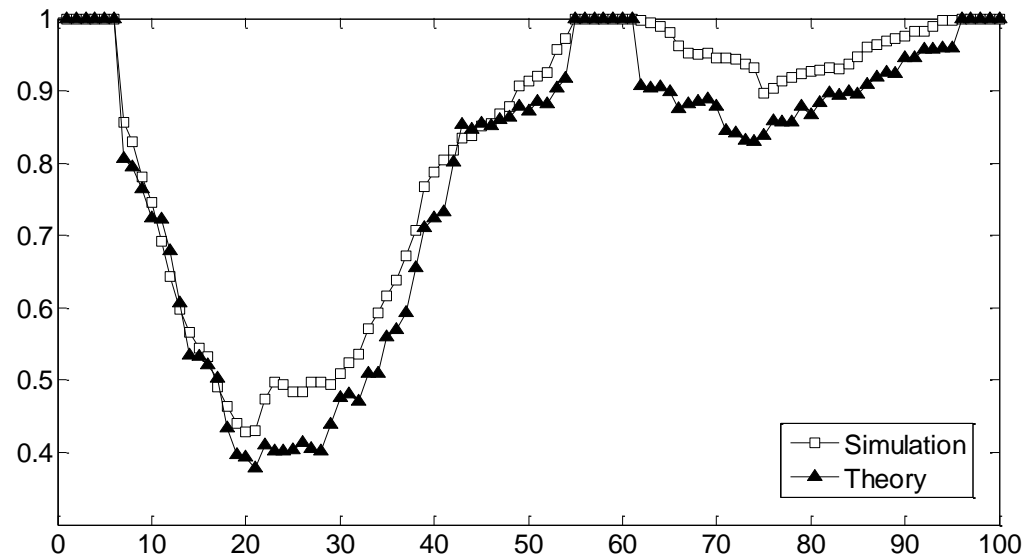

Figure 13. Numeric Result For Random Scenario 3

\section{Conclusion}

A mathematical method to predict the probability that a plane can connect to a base station in AANET is introduced in this paper. Based on our method, several future works can be done for AANET. First, design of routing protocols: Although the routing protocols of mobile ad hoc networks have already been deeply discussed in [18-20], we believe that the one based on our method may have some advantages. As we can pre-calculate the connectivity probability between a base station and a plane at each location along its airline before the plane taking off, it can be easily decided that planes on which airline are best for data transmission when the plane is at a location where several 
airlines are in its transmission radius. Moreover, since the calculation is done before the plane taking off, the number of routing maintenance packages is reduced, which means the network load is lowered. Second, sites selection of base stations: Since we have all the FCOM's extension paths from where the plane is to one of the base stations, the average hops between them can be estimated. Then we can try to find a way to set the base stations to the locations that make the number of average hops of the networks minimum. Third, security analysis: By analyzing the average hops and the paths' structure of a specified network, we can estimate the distribution of the cluster size and the structure of the clusters. Then, it is possible to find the weak points of the networks.

\section{Acknowledgements}

Our research is supported by the National Natural Science Foundation of China (Grant No. 61171089, 91438104 and 61302054), Chongqing Science \& Technology Commission (Grant No. cstc2014yykfA40002) and the 2013 Innovative Team Construction Project of Chongqing Universities.

\section{References}

[1] E. Sakhaee and A. Jamalipour, "IEEE Journal on Selected Areas in Communications", vol. 24, no. 9, (2006).

[2] Q. Vey, A. Pirovano, J. Radzik and F. Garcia, "Aeronautical Ad Hoc Network for Civil Aviation", Communication Technologies for Vehicles Lecture Notes in Computer Science, (2014).

[3] M. Schnell and S. Scalise, "IEEE Aerospace and Electronic Systems Magazine", vol. 22, no. 5, (2007).

[4] K. Karras, "Aeronautical Mobile Ad Hoc Networks", 14th European Wireless Conference, (2008).

[5] R. Justin and J. Abdul, "IEEE Transactions on Aerospace and Electronic Systems", vol. 47, no. 4, (2011).

[6] D. Medina, F. Hoffmann and S. Ayaz, "Feasibility of an aeronautical mobile ad hoc network over the north atlantic corridor", 5th Annual IEEE Communications Society Conference on Sensor, Mesh and Ad Hoc Com-munications and Networks, (2008).

[7] B. Zheng, H. Y. Zhang and P. Sun, "Journal of Shanghai Jiaotong University”, vol. 46, no. 4, (2012).

[8] T. X. M. Nguyen, Y. Miyanaga and C. Saivichit, "IEICE Transactions on Communications", E94-B, vol. 6, (2011).

[9] J. Yan, C. Hua, C. Chen and X. Guan, Wireless Networks, vol. 21, no. 3, (2014).

[10] D. Miorandi and E. Altman, Wireless Networks, vol. 12, no. 5, (2006).

[11] M. Boegsted, J. G. Rasmussen and H. P. Schwefel, Wireless Networks, vol. 17, no. 1, (2011).

[12] A. Behnad and S. N. Esfahani, IEEE Commun. Lett., vol. 14, no. 7, (2010).

[13] B. Vaidya, T. H. Kim, J. H. Park and Y. J. Lee, International Journal of Communication Systems, vol. 22, no. 29, (2009).

[14] M. Desai and D. Manjunath, IEEE Commun. Lett., vol. 6, no. 10, (2002).

[15] C. Bettstetter, The Computer Journal, vol. 47, no. 4, (2004).

[16] A. V. Babu and V. K. M. Ajeer, "International Journal of Communication Systems", vol. 26, no. 7, (2013).

[17] Z. Sheng, S. Q. Xie and C. Y. Pan, "Probability Theory and Mathematical Statistics", Hangzhou, (2010).

[18] G. Varaprasad, International Journal of Communication Systems, vol. 27, (2014).

[19] A. Esmailpour, N. Nasser and J. A. Dallal, International Journal of Communication Systems, vol. 26, (2013).

[20] L. Xie, X. Jia and K. Zhou, International Journal of Communication Systems, vol. 25, (2012). 